\title{
Description of Simulium damascenoi (Diptera: Simuliidae) Male and the Black-Fly Species from the State of Amapá, Brazil
}

\author{
Neusa Hamada/ ${ }^{+}$, Rosaly Ale-Rocha, Sérgio Luíz Bessa Luz* \\ Coordenação de Pesquisas em Entomologia, Instituto Nacional de Pesquisas da Amazônia, Caixa Postal 478, 69011-970 Manaus, \\ AM, Brasil *Centro de Pesquisa Leônidas e Maria Deane-Fiocruz, Manaus, AM, Brasil
}

Five species are included in the Simulium siolii group, which is placed in the subgenus Psaroniocompsa (Diptera: Simuliidae). Of these five species, only two (Simulium siolii Py-Daniel and Simulium tergospinosum Hamada) have been described in all their life stages, except eggs. Knowledge of the taxonomic characters of all life stages of a species is important in order to clarify interspecific and higher-level taxonomic relationships. The objectives of the present study are to describe the male of Simulium damascenoi Py-Daniel, to provide a list of black-fly species their bionomics and distributions in the state of Amapá, Brazil, and to provide an identification key for larvae and pupae for these species.

Key words: aquatic insects - Simulium damascenoi - taxonomy - species distribution - Amapá - Brazil

The group siolii is placed in the subgenus Psaroniocompsa (Diptera: Simuliidae) and has been composed, until now, of five species: Simulium siolii Py-Daniel, 1988, Simulium lourencoi Py-Daniel, 1988, Simulium damascenoi Py-Daniel, 1988, Simulium guaporense PyDaniel, 1989 and Simulium tergospinosum Hamada, 2000 (Py-Daniel 1988, 1989, Hamada 2000). Of these five species, only two have been described in all their life stages, except eggs: S. siolii and S. tergospinosum. Knowledge of the characters of all life stages is important to clarify taxonomic problems, especially in black flies where there are many species complexes.

The following species have already been reported from the state of Amapá: Simulium cauchense Floch \& Abonnenc, S. damascenoi Py-Daniel, S. goeldii Cerqueira $\&$ Nunes de Mello, Simulium guianense Wise, Simulium inaequale Paterson \& Shannon, S. incrustatum Lutz (as Simulium aequifurcatum Lutz), Simulium iracouboense Floch \& Abonnenc, Simulium maroniense Floch \& Abonnenc, Simulium oyapockense Floch \& Abonnenc, Simulium perflavum Roubaud, Simulium quadrifidum Lutz, Simulium rorotaense Floch \& Abonnenc and Simulium trombetense Hamada, Py-Daniel \& Adler (Py-Daniel 1988, Coscarón et al. 1992, 1996, Charalambous et al. 1996, Hamada \& Adler 1998, 1999). Aside from the work of Coscarón et al. (1992), no information on the distribution and bionomics of the black-fly fauna from Amapá has been provided before.

The objectives of this study are to provide: (1) the first description of $S$. damascenoi male; (2) identification

This study received partial financial support from the Third World Academy of Sciences, PPI 1-3400, 1-3070 (MCT/INPA), CNPq (grant no. 468942/00-9) and Fiocruz.

${ }^{+}$Corresponding author. Fax: +55-92-642.8909. E-mail: nhamada@inpa.gov.br.

Received 7 November 2002

Accepted 31 January 2003 keys for larvae and pupae; (3) bionomics and (4) distribution information on the black-fly fauna in the study area.

\section{MATERIALS AND METHODS}

Sampling was done in four periods, September 1993, July 1996, July 2000 and June 2001, in the state of Amapá, located in the North region of Brazil. The choice for the sampling sites was based mainly on accessibility by road, and in some cases by boat. The sampled sites lie on a transect from south to north, around the federal road (BR 156) that connects Laranjal do Jari and Oiapoque counties (Fig. 1).

Electrical conductivity (Cole-Parmer conductivimeter), $\mathrm{pH}$ (Cole-Parmer $\mathrm{pH}$ meter), temperature (alcohol thermometer), geographic position (Garmin GPS) and stream width also were estimated, whenever possible, at each sampled site.

Larvae and pupae were hand collected directly from the substrate - deciduous tree leaves, green leaves and roots of the streamside vegetation, leaves of Podostemaceae (an aquatic plant) and rocks under the water.

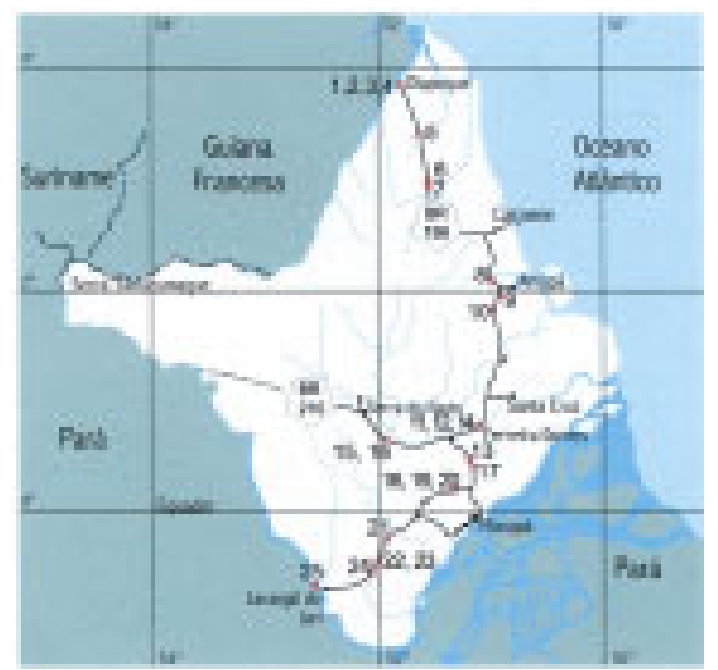

Fig. 1: map of study area, state of Amapá, Brazil, with sampled sites. Note: complete site information can be found in Table I. 
Larvae were preserved in Carnoy solution (3 parts absolute ethanol: 1 part glacial acetic acid); pupae were preserved in absolute ethanol. Some pupae were maintained alive in a glass vial with a piece of wet filter paper to obtain adults. After emergence, the adults were preserved in $80 \%$ ethanol; pharate adults that did not emerge were also preserved in $80 \%$ ethanol. All specimens were preserved on ice in the field; in the laboratory they were maintained refrigerated at $4^{\circ} \mathrm{C}$. In the laboratory, adults in $80 \%$ ethanol were dehydrated according to the technique of Sabrosky (1966) and pinned. Permanent slides were mounted using Euparal ${ }^{\mathbb{R}}$ as the mounting medium after being clarified in hot $95 \%$ lactic acid.

All specimens used in this study are deposited in the Invertebrate Collection of the Instituto Nacional de Pesquisas da Amazônia (Inpa), Manaus, AM, Brazil.

\section{RESULTS AND DISCUSSION}

\section{Taxonomy \\ Simulium (Psaroniocompsa) damascenoi Py-Daniel} (Figs 2-17)

Simulium (Psaroniocompsa) damascenoi Py-Daniel, 1988:304, Figs 69-97.

Male description

Body length, $1.65 \mathrm{~mm}(\mathrm{n}=4)$. Wing length, $1.42 \mathrm{~mm}(\mathrm{n}=$ 4). Lateral thorax length $0.4 \mathrm{~mm}(\mathrm{n}=4)$. Antenna $0.4 \mathrm{~mm}$ $(n=5)$ long, dark brown, except the scape, pedicel and half distal region of the first flagellomere, yellow (Fig. 3). Maxillary palpus brown (Fig. 2), sensory vesicle almost one-third of the size of palpomere that holds it. Scutum black, with two antero-lateral quadrangular spots, with irregular posterior region (Figs 16,17). Anepisternum and katepisternum brown with silver pruinosity; scutellum dark brown with brown hairs that have golden highlights, postnotum brown with silver pubescence. Femur and tibiae with scale-like setae distributed with filiform setae ones. Abdominal sclerites brown; membranous areas brown; basal fringe hair brown. Tergites dark brown; gonocoxite and gonostylus brown (Figs 7, 8), gonostylus bearing one apical tooth and one longitudinal ridge; ventral plate as in Fig. 4; median sclerite oval (Fig. 5); paramere with spines (Fig. 6).

Simulium damascenoi female - Additional taxonomic information

Body length, $1.83 \mathrm{~mm}(\mathrm{n}=2)$. Wing length, $1.65 \mathrm{~mm}(\mathrm{n}=1)$. Lateral thorax length $0.43 \mathrm{~mm}(\mathrm{n}=2)$. Female was described from a specimen dissected from its pupal skin (Py Daniel 1988); therefore, no information on scutum pattern was provided. Scutum with paired thick silver stripes (Figs 14, 15). Distal abdominal tergites (VI-IX) striking distinct from the others, pale, greenish in color, with a varnish-like appearance (Fig. 13), tergites III-V brown, with median plate varnish-like in appearance, tergites 1-2 brown with silver pruinosity. Cibarium (Fig. 11) has typical Psaroniocompsa subgenus configuration; sensory vesicle of maxillary palpus about a half of basal article length as shown in Fig. 9; antenna color pattern as in Fig. 10. Spermatheca subspherical, with cuticular microspines, spermatheca duct and area of attachment not pigmented (Fig. 12).

\section{S. damascenoi specimens examined}

Brazil, state of Amapá: Igarapé Davi Grande, Amapá county, 24/vii/2000, Hamada N, Barbosa UC, Cavalcante A, Freire R (Inpa), 1 pharate male (thorax and part of abdomen pinned, head and genitalia in slide mount), 1 pharate female in slide mount; 18/vi/2001, Hamada N, Ale-Rocha R, Freire R (Inpa), 4 pinned male, 2 pinned female, 1 pharate male (thorax pinned, head and abdomen in slide mount); Igarapé Água Branca, near Carnot Village, 25/vii/2000, Hamada N, Barbosa UC, Cavalcante A, Freire R (Inpa), 1 pinned male, 1 pinned female; 19/vi/2001, Hamada N, AleRocha R, Freire R (Inpa), 1 pharate female in slide mount; Igarapé do Henrique, 18/vi/2001, Hamada N, Ale-Rocha R, Freire R (Inpa), 1 pharate male (head and thorax pinned, abdomen in slide mount), 1 pharate male in slide mount.

\section{Remarks}

At the male stage, $S$. damascenoi can be distinguished from $S$. siolii and $S$. tergospinosum (the only species for which the males are known in the siolii group) by the presence of the paired quadrangular spot in the anterolateral region of the scutum. Females of this species can be distinguished from the other known females in the siolii group (S. siolii and S. tergospinosum) by the pale, greenish color of the distal abdominal tergites (VI-IX), which is strikingly distinct from the dark brown color of these structures in the other species. Another difference is that the thick-paired bands on the $S$. damascenoi female scutum are of similar width, while in $S$. tergospinosum and $S$. siolii female scutum, the thick-paired bands turn narrower in the distal region.

Identification key for Simulium spp. (Diptera: Simuliidae) larvae in The State of Amapá, Brazil

1. Larva without ventral posterior papillae ....................... 2

1 '. Larva with ventral posterior papillae .......................... 10

2. Subesophageal ganglion pigmented .......................... 3

2'. Subesophageal ganglion not pigmented ..................... 6

3. Body with dorsal subtriangular protuberances, which increase in length in the antero-posterior direction in the first five abdominal segments, dissected gill histoblast with 4 filaments ................................... S. damascenoi 3'. Body without dorsal subtriagular protuberances, dissected gill histoblast with 8 or more filaments ................ 4 4. Dissected gill histoblast with 8 filaments, dorsal side of terminal region of abdomen without racket-like shape setae ................................................. S. subpallidum 4'. Dissected gill histoblast with 12 filaments, dorsal side of terminal region of abdomen with racket-like shape setae ................................................................... 5 5. Larvae of median size (length 4- $6 \mathrm{~mm}$ ); terminal region of abdomen not truncated .................... S. guianense s.l. 5'. Larvae large (length $7.3-8.8 \mathrm{~mm}$ ); terminal region of abdomen truncated ............................................ S. hirtipupa 6. Larvae small to medium size $(<5.5 \mathrm{~mm})$; cephalic capsule, dorsally, with dark pattern in the form of a circle with crenate outer border; dissected histoblast with 8 filaments ....................................... S. iracouboense 6'. Larvae larger (>6 mm); cephalic capsule, dorsally, without defined pattern; dissected histoblast with 8 or more filaments

7. Gill histoblast in situ large $(>1.5 \mathrm{~mm})$ 


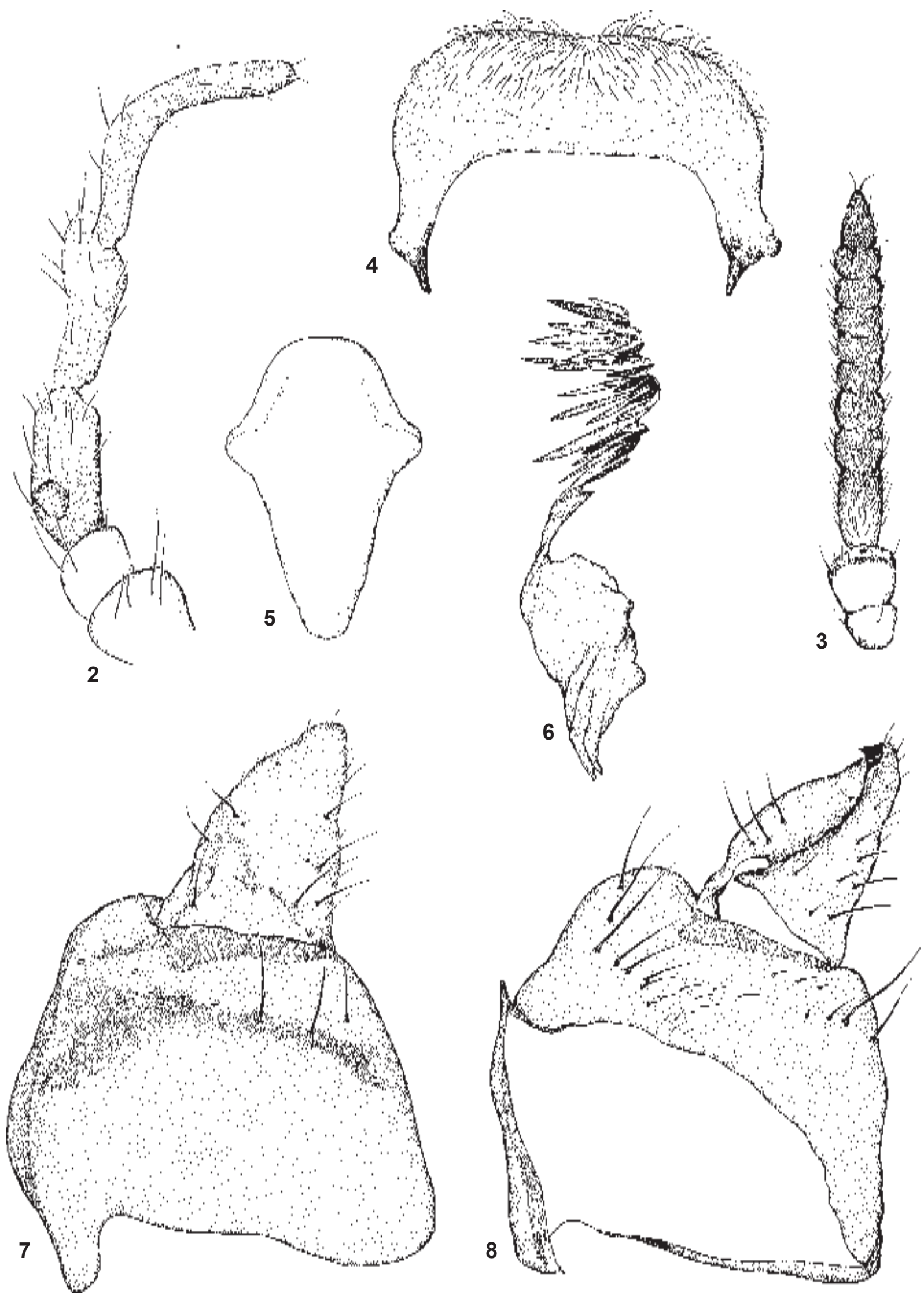

Male of Simulium damascenoi (Diptera: Simuliidae). Fig. 2: maxillary palpus. Fig. 3: antenna. Fig. 4: ventral plate. Fig. 5: median sclerite. Fig. 6: paramere. Gonocoxite and gonostylus. Fig. 7: ventral view. Fig. 8: dorsal view 

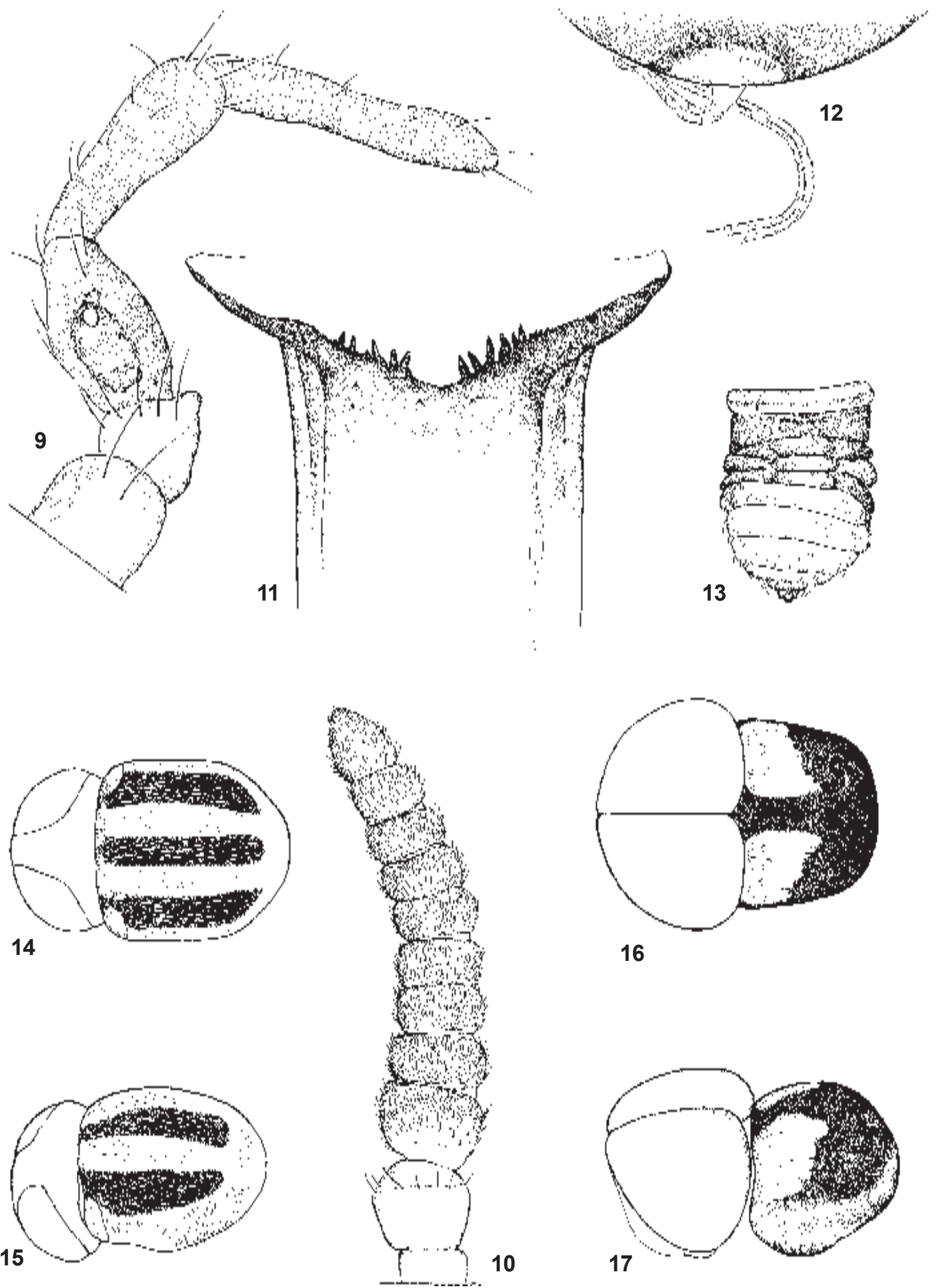

16

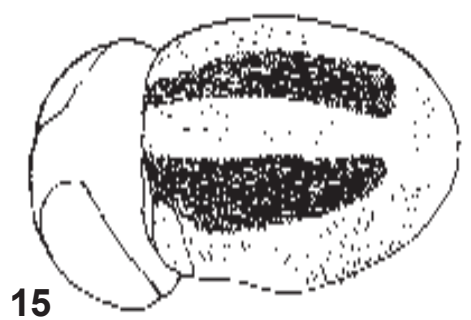

10



Simulium damascenoi (Diptera: Simuliidae). Female. Fig. 9: maxillary palpus. Fig. 10: antenna. Fig. 11: cibarium. Fig. 12: spermatheca. Fig. 13: abdomen. Fig. 14: scutum, dorsal view. Fig. 15: scutum, dorso-lateral view. Male. Fig. 16: scutum, dorsal view. Fig. 17: scutum, dorso-lateral view 
7'. Gill histoblast in situ not large $(<1.5 \mathrm{~mm})$ 9 8. Larvae with coloration dark green; gill histoblast in situ with numerous thin filaments coming out from thick trunk; dissected gill histoblast with more than 100 filaments ...... S. trombetense 8'. Larvae with coloration pale green; gill histoblast in situ with thick filaments visible; dissected gill histoblast with 8 filaments S. perflavum 9. Dissected gill histoblast with 18-21 thick, dark filaments branching near base, with dorsal filaments shorter than ventral ones; in anterior view, filaments forming open rosette basally .. S. maroniense 9'. Dissected gill histoblast with 18-21 thinner, lightly pigmented filaments, varying in size and branching pattern; in anterior view filaments projected forward, not forming open rosette basally S. rorotaense 10. Subesophageal ganglion pigmented; dissected gill histoblast with 6 filaments ... 11 10'. Subesophageal ganglion not pigmented; dissected gill histoblast with number of filaments variable ............ 12 11. Postgenal cleft longer than wide, constricted at base, so that maximum width is about one-third of length; postgenal bridge between one-half and one-third as long as hypostomium .... S. incrustatum 11'. Postgenal cleft approximately as long as wide at the broadest point, narrowed at base; postgenal bridge half as long as hypostomium ...................... S. oyapockense s.l. 12. Distal portion of postgenal cleft pointed; dissected gill histoblast with 6 filaments .......................... S. inaequale 12'. Distal portion of postgenal cleft rounded; dissected gill histoblast with 4 or 8 filaments ... 13 13. Postgenal cleft not wide at the distal portion; lateral mandibular process bifid or trifid; second antenal article larger than the first; dissected gill histoblast with 8 filaments S. goeldii 13'. Postgenal cleft wide at the distal portion; lateral mandibular process simple; second antenal article smaller than the first, dissected gill histoblast with 4 filaments ........ 14 14. Dorsal abdominal region with multiramous setae, with irregular branching pattern (Fig. 18); dissected gill histoblast with filaments branching far away from the base ....................................................... S. cauchense 14'. Dorsal abdominal region with multiramous setae, branching near the base (Fig.19); dissected gill histoblast branching near the base S. quadrifidum

Identification key for Simulium spp. (Diptera: Simuliidae) pupae in the state of Amapá, Brazil

1. Gill with more than 17 filaments 13

1'. Gill with 4-12 filaments

2. Gill with 4 filaments, cocoon slipper-shaped ................. 3

2'.Gill with 6-12 filaments, cocoon variable ..................... 5

3. Cephalic plate with pointed tubercles; pattern of gill filaments variable, cocoon with proeminent dorsal projection ....

3'. Cephalic plate without tubercles; base of gill filaments very short, with 2 principal trunks, cocoon without proeminent dorsal projection ... S. damascenoi 4. Primary branch of gill filaments branching at more than half of total length of filament ........................... S. cauchense 4'. Primary branch of gill filaments branching near base S. quadrifidum

5. Gill with 6 filaments ........................................................... 6

5'.Gill with 8-12 filaments .................................................. 8

6. Gill filaments short, approximately the length of pupae . S. oyapockense s.l.

6'. Gill filaments long, at least $1.5 \mathrm{x}$ the length of pupa .... 7 7. Gill filaments branching near base (primary and secondary branches very short). S. inaequale 7'. Gill filaments branching at variable distances from base S. incrustatum 8. Gill with 12 filaments; gill filaments very short, shorter than body length, cocoon shoe-shaped ........................... 9 8 '. Gill with 8 filaments, as long as the length of the body, cocoon slipper-shaped ..................................................... 10 9. Cephlalic plate and exposed portion of the thorax without long setae ............................... S. guianense s.l. 9'. Cephalic plate and exposed portion of the thorax with numerous long setae ......................... S. hirtipupa 10. Cocoon with dorsal median projection; gill filaments $1.5 \mathrm{x}$ or more the body length; gill filaments branching at different distances from base .............................. S. goeldii 10 '. Cocoon without dorsal median projection; gill filaments shorter than $1.5 \mathrm{x}$ the body length; gill filaments branching near base; if not, gill filaments shorter than cocoon; gill filaments branching at same or different heights .

... 11

11. Gill filaments branching from one common trunk, near base and usually at same heights .................... S. perflavum 11'. Gill filaments branching at different heights; near base, main trunk branching in three primary branches ............ 12 12. Cephalic trichomes short and simple ... S. iracouboense 12'. Cephalic trichomes with 2-5 branches .. S. subpallidum 13. Gill with more than 100 filaments ......... S. trombetense 13 '. Gill with 17-23 filaments 14 14. Gill with thin, lightly pigmented filaments, varying in size and branching pattern; in anterior view, filaments projected forward, not forming rosette ........... S. rorotaense 14 '. Gill with thick, darkly pigmented filaments; dorsal filaments shorter than ventral ones; in anterior view, filaments forming open rosette basally .... S. maroniense

\section{Bionomics and distribution}

Sampling was done in 25 streams or rivers (Table I, Fig. 1) including areas with savanna and forest type of vegetation. Streams and rivers sampled in the study area had width varying from 2 to $500 \mathrm{~m}, \mathrm{pH}$ varying from 4.7 to 7.6 , electrical conductivity varying from below 10 to 20 $\mu \mathrm{S} / \mathrm{cm}$ and temperature ranging from 24 to $28^{\circ} \mathrm{C}$. Of the 25 streams sampled, 19 had bedrock, boulders or small stones while only six had sandy/clayey streambeds. Many of the streams with rocks $(n=14)$ had plants belonging to the family Podostemaceae, which are frequently used as substrates by many different aquatic insects, including black-fly larvae and pupae.

Fifteen black-fly species were collected during the sampling period (Tables I, II). This is the first record of the species Simulium hirtipupa Lutz and Simulium subpallidum Lutz in Amapá.

The mean number of species per collection was $2.5( \pm$ 1.19) $(\mathrm{n}=39$, range $=1$ to $5 \mathrm{spp}$.). This is similar to the mean number of black-fly species found in streams in 
TABLE I

Simulium spp. (Diptera: Simuliidae) collection data in the state of Amapá, Brazil

\begin{tabular}{|c|c|c|c|c|}
\hline $\begin{array}{l}\text { Collectio } \\
\text { number }\end{array}$ & Collection location & Date & Collectors & $\begin{array}{l}\text { Collected } \\
\text { species }\end{array}$ \\
\hline 1 & Oiapoque river, $03^{\circ} 48^{\prime} \mathrm{N} 51^{\circ} 53^{\prime} \mathrm{W}$ & $\begin{array}{l}26.07 .2000 \\
20.06 .2001\end{array}$ & $\begin{array}{l}\text { NH, UCB, AC, RF } \\
\text { NH, RAR, RF }\end{array}$ & $\begin{array}{l}1,2,3,9 \\
1,2\end{array}$ \\
\hline 2 & Pantanary stream, Oiapoque county, $03^{\circ} 47^{\prime} \mathrm{N} 51^{\circ} 48^{\prime} \mathrm{W}$ & 20.06 .2001 & NH, RAR, RF & 1,8 \\
\hline 3 & $\begin{array}{l}\text { Stream near airport, Oiapoque county, } 03^{\circ} 52^{\prime} \mathrm{N} 51^{\circ} 47^{\prime} \mathrm{W} \\
\text { Stream near entrance of Oiapoque city, (owner - Mr Edmilson)BR } 156\end{array}$ & $\begin{array}{l}25.07 .1996 \\
28.07 .2000 \\
27.07 .1996\end{array}$ & $\begin{array}{l}\text { NH, LA } \\
\text { NH, UCB, AC, RF } \\
\text { NH, LA }\end{array}$ & $\begin{array}{l}6,8 \\
6,8 \\
8,9\end{array}$ \\
\hline 5 & $\begin{array}{l}\text { Stream near Primeiro do Caciporé, BR 156, } 90 \mathrm{~km} \text { from } \\
\text { Oiapoque city, } 03^{\circ} 11^{\prime} \mathrm{N} 51^{\circ} 32^{\prime} \mathrm{W}\end{array}$ & 27.07.2000 & $\mathrm{NH}, \mathrm{UCB}, \mathrm{AC}, \mathrm{RF}$ & $5,8,9,12$ \\
\hline 6 & $\begin{array}{l}\text { Água Branca stream, near Carnot village, BR } 156 \\
\text { Macapá-Oiapoque, } 02^{\circ} 40^{\prime} \mathrm{N} 51^{\circ} 21^{\prime} \mathrm{W}\end{array}$ & $\begin{array}{l}25.07 .1996 \\
28.07 .2000 \\
19.06 .2001\end{array}$ & $\begin{array}{l}\text { NH, LA } \\
\text { NH, UCB, AC, RF } \\
\text { NH, RAR, RF }\end{array}$ & $\begin{array}{l}5,8,9,12,14 \\
5,8,9,12,14 \\
1,5,8,9,12\end{array}$ \\
\hline 7 & $\begin{array}{l}\text { Torrão stream, near Água Branca stream, BR } 156 \\
\text { Macapá-Oiapoque, } 02^{\circ} 27^{\prime} \mathrm{N} 51^{\circ} 13^{\prime} \mathrm{W}\end{array}$ & $\begin{array}{l}25.07 .1996 \\
28.07 .2000 \\
19.06 .2001\end{array}$ & $\begin{array}{l}\text { NH, LA } \\
\text { NH, UCB, AC, RF } \\
\text { NH, RAR, RF }\end{array}$ & $\begin{array}{l}5,8,9 \\
8,9 \\
8,9\end{array}$ \\
\hline 8 & $\begin{array}{l}\text { Cachoeira Grande river, Amapá county, BR } 156 \\
\text { Macapá-Oiapoque, } 02^{\circ} 09^{\prime} \mathrm{N} 50^{\circ} 55^{\prime} \mathrm{W}\end{array}$ & $\begin{array}{l}28.09 .1993 \\
27.07 .2000\end{array}$ & $\begin{array}{l}\mathrm{NH} \\
\mathrm{NH}, \mathrm{UCB}, \mathrm{AC}, \mathrm{RF}\end{array}$ & $\begin{array}{l}1,3,12 \\
1,2,12\end{array}$ \\
\hline 9 & $\begin{array}{l}\text { Davi Grande stream, Amapá county, BR } 156 \\
\text { Macapá-Oiapoque, } 01^{\circ} 55^{\prime} \mathrm{N} 50^{\circ} 51^{\prime} \mathrm{W}\end{array}$ & $\begin{array}{l}08.1993 \\
25.07 .1996 \\
24.07 .2000 \\
18.06 .2001\end{array}$ & $\begin{array}{l}\text { NH } \\
\text { NH, LA } \\
\text { NH, UCB, AC, RF } \\
\text { NH, RAR, RF }\end{array}$ & $\begin{array}{l}12 \\
8 \\
8,9,12 \\
8,12\end{array}$ \\
\hline 10 & $\begin{array}{l}\text { Henrique stream, Amapá County, BR } 156 \\
\text { Macapá-Oiapoque, } 01^{\circ} 45^{\prime} \mathrm{N} 50^{\circ} 52^{\prime} \mathrm{W}\end{array}$ & $\begin{array}{l}25.07 .1996 \\
28.07 .2000 \\
18.06 .2001\end{array}$ & $\begin{array}{l}\text { NH, LA } \\
\text { NH, UCB, AC, RF } \\
\text { NH, RAR, RF }\end{array}$ & $\begin{array}{l}8 \\
9,12 \\
8,12\end{array}$ \\
\hline 11 & $\begin{array}{l}\text { Araguari river, BR } 156 \text { Macapá-Oiapoque, Ferreira Pena } \\
\text { bridge, } 00^{\circ} 51^{\prime} \mathrm{N} 51^{\circ} 11^{\prime} \mathrm{W}\end{array}$ & 28.09.1993 & $\mathrm{NH}$ & 1 \\
\hline 12 & $\begin{array}{l}\text { Stream tributary of Araguari river, above Ferreira Pena } \\
\text { bridge, } 00^{\circ} 51^{\prime} \mathrm{N} 51^{\circ} 11^{\prime} \mathrm{W}\end{array}$ & $\begin{array}{l}28.09 .1993 \\
18.06 .2001\end{array}$ & $\begin{array}{l}\text { NH } \\
\text { NH, RAR, RF }\end{array}$ & $\begin{array}{l}7,10 \\
7,10\end{array}$ \\
\hline 13 & $\begin{array}{l}\text { Flexal stream, Araguari tributary, Monte Taboa, BR } 156 \\
\text { Macapá-Oiapoque, } 00^{\circ} 29^{\prime} \mathrm{N} 51^{\circ} 07^{\prime} \mathrm{W}\end{array}$ & 24.07.2000 & $\mathrm{NH}, \mathrm{UCB}, \mathrm{AC}, \mathrm{RF}$ & $7,8,11$ \\
\hline 14 & $\begin{array}{l}\text { Stream near Ferreira Gomes village, road to UHE Paredão, } \\
00^{\circ} 50^{\prime} \mathrm{N} 51^{\circ} 11^{\prime} \mathrm{W}\end{array}$ & 30.07 .1996 & $\mathrm{NH}, \mathrm{LA}$ & $7,8,9,14$ \\
\hline 15 & $\begin{array}{l}\text { Água Fria river, Macapá-Serra no Navio road, large bridge, } \\
00^{\circ} 48^{\prime} \mathrm{N} 51^{\circ} 58^{\prime} \mathrm{W}\end{array}$ & 29.07.1996 & $\mathrm{NH}, \mathrm{LA}$ & $1,2,9$ \\
\hline 16 & $\begin{array}{l}\text { Stream near Serra do Navio village, Cachaço stream tributary, } \\
\sim 00^{\circ} 48^{\prime} \mathrm{N} 51^{\circ} 58^{\prime} \mathrm{W}\end{array}$ & 29.07.1996 & NH, LA & $5,7,8,9$ \\
\hline 17 & $\begin{array}{l}\text { Stream near EMBRAPA, BR } 156 \text { Macapá-Oiapoque, } \\
\left.00^{\circ} 28^{\prime} \mathrm{N} 51^{\circ} 06^{\prime} \mathrm{W} 6\right)\end{array}$ & 30.07 .1996 & $\mathrm{NH}, \mathrm{LA}$ & 8,11 \\
\hline 18 & $\begin{array}{l}\text { Stream Cachoeirinha (balneário), Macapá county, BR } 156 \\
\text { Macapá-Jari, } 00^{\circ} 12^{\prime} \mathrm{N} 51^{\circ} 28^{\prime} \mathrm{W}\end{array}$ & 24.07.1996 & $\mathrm{NH}, \mathrm{LA}$ & 7,9 \\
\hline 19 & $\begin{array}{l}\text { Stream } \sim 19 \mathrm{~km} \text { after stream Cachoeirinha, Macapá county, } \\
\text { BR } 156 \text { Macapá-Jari, } 00^{\circ} 12^{\prime} \mathrm{N} 51^{\circ} 28^{\prime} \mathrm{W}\end{array}$ & 24.07.1996 & NH, LA & $7,9,13$ \\
\hline 20 & $\begin{array}{l}\text { Stream } 24 \text { km after stream Cachoeirinha, Macapá county, } \\
\text { BR } 156 \text { Macapá-Jari, } 00^{\circ} 12^{\prime} \mathrm{N} 51^{\circ} 28^{\prime} \mathrm{W}\end{array}$ & 24.07.1996 & $\mathrm{NH}, \mathrm{LA}$ & 7,11 \\
\hline 21 & Stream on BR 156, Macapá-Jari, $00^{\circ} 05^{\prime} \mathrm{S} 51^{\circ} 43^{\prime} \mathrm{W}$ & 31.07 .1996 & NH, LA & 7 \\
\hline 22 & Stream on BR 156 , Macapá-Jari, $00^{\circ} 27^{\prime} \mathrm{S} 52^{\circ} 05^{\prime} \mathrm{W}$ & $\begin{array}{l}31.07 .1996 \\
23.06 .2001\end{array}$ & $\begin{array}{l}\text { NH, LA } \\
\text { NH, RAR, RF }\end{array}$ & $\begin{array}{l}4,7,8,14 \\
8,14\end{array}$ \\
\hline 23 & Stream on BR 156 , Macapá-Jari, $00^{\circ} 27^{\prime} \mathrm{S} 52^{\circ} 07^{\prime} \mathrm{W}$ & 31.07 .1996 & $\mathrm{NH}, \mathrm{LA}$ & $4 / 5,7,14$ \\
\hline 24 & Stream at Marinho village. On the road to Laranjal do Jari & 31.07 .1996 & NH, LA & 8 \\
\hline 25 & $\begin{array}{l}\text { Jari river, Santo Antônio waterfall, Laranjal do Jari county, } \\
00^{\circ} 39^{\prime} \mathrm{S} 52^{\circ} 30^{\prime} \mathrm{W}\end{array}$ & 24.06 .2001 & $\mathrm{NH}, \mathrm{RAR}, \mathrm{RF}$ & 15 \\
\hline
\end{tabular}

Date: day,month, year; BR: Federal road; UHE: hydroelectric dam; LA: Luís Aquino; NH: Neusa Hamada; UCB: Ulysses C Barbosa; RF: Raimundo Freire; AC: Aracy Cavalcante; RAR: Rosaly Ale-Rocha; Species - 1: S. iracouboense; 2: S. guianense (s.l.); 3: S. oyapockense (s.l.); 4: S. rorotaense; 5: S. maroniense, 6: S. trombetense, 7: S. perflavum; 8: S. quadrifidum; 9: S. cauchense; 10: S. inaequale; 11: S. incrustatum; 12: S. damascenoi; 13: S. goeldii; 14: S. subpallidum; 15: S. hirtipupa 
TABLE II

Habitat characterization of Simulium spp. (Diptera: Simuliidae) collected in the state of Amapá, Brazil

\begin{tabular}{|c|c|c|c|c|c|c|c|c|}
\hline Species & $\begin{array}{l}\text { Occurrence } \\
\text { per site }\end{array}$ & $\begin{array}{c}\text { Habitat } \\
\text { width }(\mathrm{m})\end{array}$ & $\begin{array}{c}\text { Electrical } \\
\text { conductivity } \\
(\mathrm{mS} / \mathrm{cm})\end{array}$ & $\begin{array}{l}\text { Temperature } \\
\left({ }^{\circ} \mathrm{C}\right)\end{array}$ & $\mathrm{pH}$ & Streambed & $\begin{array}{c}\text { Vegetation } \\
\text { type } \\
\text { (number of sites) }\end{array}$ & Substrate \\
\hline S. cauchense & 12 & $5-500$ & $<20$ & $24-28$ & $4.7-6.9$ & Rock & Forest (6) / Savanna (6) & $\mathrm{D}, \mathrm{G}, \mathrm{R}, \mathrm{P}$ \\
\hline S. damascenoi & 5 & $8-100$ & $<10$ & $25-27$ & $5.5-6.4$ & Rock & Forest (2) / Savanna (3) & $\mathrm{D}, \mathrm{G}, \mathrm{R}, \mathrm{P}$ \\
\hline S. goeldii & 1 & 5 & $<10$ & 27 & 4.7 & Stones & Savanna & $\mathrm{D}$ \\
\hline S. guianense & 3 & $95-500$ & $<20$ & $24-28$ & $5.9-6.9$ & Rock & Forest (1) / Savanna (2) & $\mathrm{P}$ \\
\hline S. hirtipupa & 1 & 150 & $<20$ & 26 & 7.6 & Rock & Forest & $\mathrm{P}$ \\
\hline S. inaequale & 1 & 10 & $<10$ & 26 & 5.7 & Sand & Savanna & $\mathrm{D}$ \\
\hline S. incrustatum & 3 & $2-8.5$ & $<10$ & $25-28$ & $4.7-4.9$ & Sand/clay & Savanna & $\mathrm{D}, \mathrm{G}$ \\
\hline S. iracouboense & 6 & $40-500$ & $<10$ & $24-28$ & $5.9-6.9$ & Rock & Forest (3) / Savanna (3 & $\mathrm{D}, \mathrm{G}, \mathrm{R} ., \mathrm{P}$ \\
\hline S. maroniense & 4 & $8-17$ & $<10$ & 25 & $5.3-6.4$ & Rock & Forest & $\mathrm{D}, \mathrm{G}, \mathrm{P}$ \\
\hline S. oyapockense & 2 & $100-200$ & $<10$ & $27-28$ & $6.4-6.9$ & Rock & Forest (1) / Savanna (1) & $\mathrm{D}, \mathrm{P}$ \\
\hline S. perflavum & 10 & $3-10$ & $<20$ & $25-28$ & $4.7-5.7$ & Sand & Forest (1) / Savanna (9) & $\mathrm{D}, \mathrm{G}, \mathrm{R}$ \\
\hline S. quadrifidum & 14 & $2-40$ & $<20$ & $24-26$ & $4.9-6.4$ & Rock & Forest (8) / Savanna (6) & $\mathrm{D}, \mathrm{G}, \mathrm{R}, \mathrm{P}$ \\
\hline S. rorotaense & 1 & 10 & $<10$ & 27 & 4.7 & Rock & Savanna & $\mathrm{D}, \mathrm{P}$ \\
\hline S. subpallidum & 4 & $2-10$ & $<20$ & $25-28$ & $5.0-5.7$ & Rock & Forest (1) / Savanna (3) & $\mathrm{D}, \mathrm{G}, \mathrm{P}$ \\
\hline S. trombetense & 1 & 4 & a & 25 & 5.6 & Rock & Forest & $\mathrm{D}, \mathrm{G}, \mathrm{R}, \mathrm{Rk}$ \\
\hline
\end{tabular}

a: not measured; Rock: rock bottom/boulder; Stones: small stones; D: deciduous leaves; G: green leaves of streamside vegetation; R: roots of streamside vegetation; P: Podostemacea leaves; Rk: rock
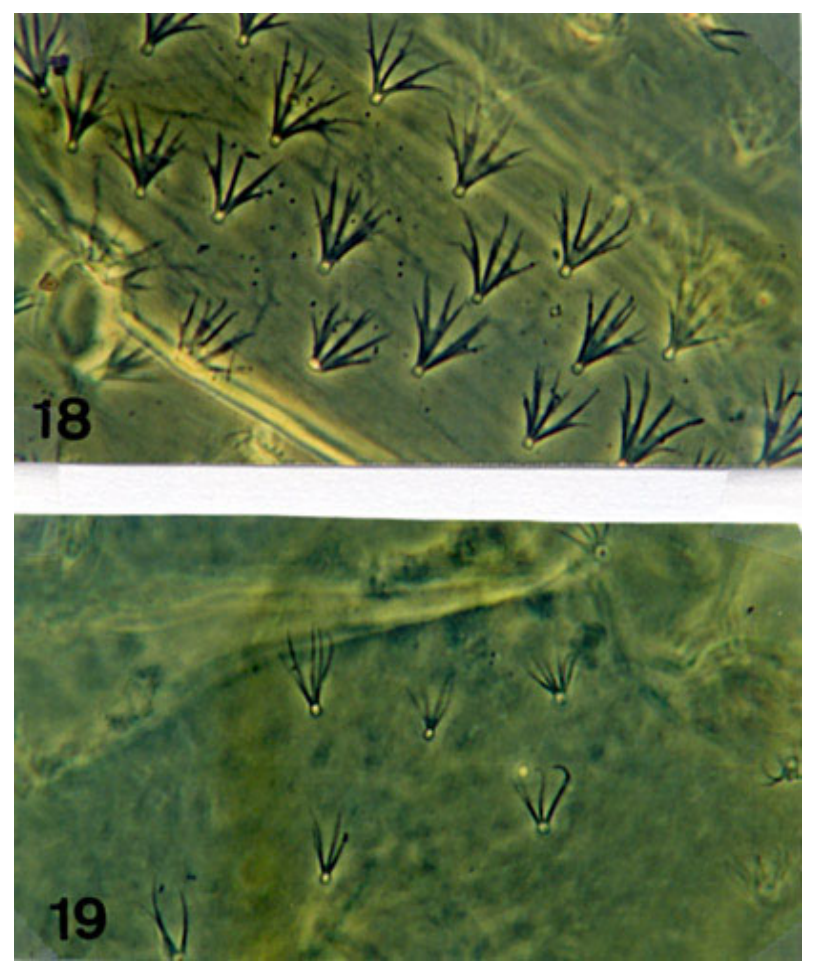

Larval abdominal dorsal setae. Fig. 18: Simulium cauchense. Fig. 19: Simulium quadrifidum

Central Amazonia by Hamada et al. (2002).

Species with high frequency, occurring in 40 to $56 \%$ of the collections, were $S$. quadrifidum, $S$. cauchense and $S$. perflavum (Table I). S. iracouboense, S. damascenoi, $S$. maroniense and S. subpallidum occurred in $16-24 \%$ of the sites and $S$. guianense, $S$. incrustatum, $S$. oyapockense s.1., S. rorotaense, $S$. trombetense, $S$. inaequale, S. goeldii and S. hirtipupa occurred in $4-12 \%$ of the sites (Table I).
Some physical-chemical characteristics of the habitat of each species sampled during this study are shown in Table II. Co-occurrences of species can be observed in Table I.

\section{ACKNOWLEDGEMENTS}

To the support provided by the Instituto Estadual de Pesquisas do Amapá-Iepa (Antônio C Farias) and Funasa, Amapá (Raimundo Freire). Luís Aquino, Raimundo Freire, Ulysses C Barbosa (Inpa), Walter L Chaves (Iepa), and Aracy Cavalcante (Funasa/Manaus) helped in the fieldwork. Deyse Cristina Queiróz Silva drew the illustrations. Philip M Fearnside reviewed the manuscript.

\section{REFERENCES}

Charalambous M, Shelley AJ, Maia Herzog M, Luna Dias APA 1996. Four new cytotypes of the onchocerciasis vector blackfly Simulium guianense in Brazil. Med Vet Entomol 10: 111-120.

Coscarón S, Cerqueira RL, Schumaker TTS, La Salvia Filho V 1992. Nuevos datos sobre distribución de simulideos de Brasil y descripción de Simulium (Coscaroniellum) cerradense sp. n. (Diptera: Simuliidae). Revta Bras Entomol 36: 111-119.

Coscarón S, Ibañez-Bernal S, Coscarón-Arias CL 1996. Revision de Simulium (Psilopelmia) Enderlein em la region Neotropical y analisis cladistico de sus especies (Diptera: Simuliidae). Acta Zool Mex 69: 37-104.

Hamada N 2000. Simulium (Psaroniocompsa) tergospinosum new species (Diptera: Simuliidae) in siolii group from the southern part of the State of Amazonas, Brazil. Mem Inst Oswaldo Cruz 95: 819-828.

Hamada N, Adler PH 1998. Taxonomy of the Simulium perflavum species-group (Diptera: Simuliidae), with description of a new species from Brazil. Insecta Mundi 12: 207226.

Hamada N, Adler PH 1999. Cytotaxonomy of four species in the Simulium perflavum species-group (Diptera: Simuliidae) from Brazilian Amazonia. Syst Entomol 24: 273-288.

Hamada N, McCreadie JW, Adler PH 2002. Species richness 
and spatial distribution of blackflies (Diptera: Simuliidae) in streams of Central Amazonia, Brazil. Freshwater Biol 47: $31-40$.

Py-Daniel V 1988. Simuliidae (Diptera: Culicomorpha) no Brasil VI. Sobre Simulium (Psaroniocompsa) siolii sp.n., Simulium (P.) lourencoi sp.n. e Simulium (P.) damascenoi sp.n.
Rev Saúde Púb 22: 292-310.

Py-Daniel V 1989. Simuliidae (Diptera: Culicomorpha) no Brasil X. Descrição de Simulium (Psaroniocompsa) guaporense sp.n. Rev Saúde Púb 23: 502-508.

Sabrosky CW 1966. Mounting insects from alcohol. Bull Am Entomol Soc 12: 349. 